\title{
The dynamic effect in capillary pressure during the displacement process in ultra-low permeability sandstone reservoirs
}

\author{
Ying $\mathrm{Li}^{1,2}{ }^{*}$, Haitao $\mathrm{Li}^{1}$, Jianchao $\mathrm{Cai}^{3}$, Qirui $\mathrm{Ma}^{1}$, Jianfeng Zhang ${ }^{4}$ \\ ${ }^{1}$ State Key Laboratory of Oil and Gas Reservoir Geology and Exploitation, Southwest Petroleum University, Chengdu 610500, P. R. China \\ ${ }^{2}$ Postdoctoral Research Station, Southwest Petroleum University, Chengdu 610500, P. R. China \\ ${ }^{3}$ Institute of Geophysics and Geomatics, China University of Geosciences, Wuhan 430074, P. R. China \\ ${ }^{4}$ The Second Gas Production Plant, Sinopec Northwest Oil and Gas Company, Nanchong 637400, P. R. China
}

\section{Keywords:}

Ultra-low permeability

displacement process

dynamic effect

multiphase flow

sandstone

Cited as:

Li, Y., Li, H., Cai, J., Ma, Q., Zhang, J.

The dynamic effect in capillary pressure

during the displacement process in

ultra-low permeability sandstone

reservoirs. Capillarity, 2018, 1(2): 11-18, doi: 10.26804/capi.2018.02.01.

\begin{abstract}
:
The relationship between the capillary pressure and saturation is a primary factor to describe and simulate the multiphase flow. This relationship is also fundamental to understand the characteristics of oil and gas reservoirs and make the reservoir development plan. Traditionally, the capillary pressure is measured under the equilibrium process; however, this equilibrium is hard to establish when the multiphase flow is expected in low to tight permeability porous media, and the capillary pressure is dynamic. This laboratory study conducts specially designed dynamic displacement experiments to examine the dynamic effect in capillary pressure in ultra-low permeability sandstone oil reservoirs. The dynamic capillary pressure, the dynamic relative permeabilities, the dynamic coefficient and the change rate of water saturation are obtained. Results show that the dynamic coefficient is relatively larger in ultra-low permeability reservoirs compared with that in high to low permeability reservoirs. Difference between the dynamic and the steady capillary pressures becomes more significant for less permeable porous media, with a higher dynamic coefficient and a stronger dynamic effect. Wettability advancement has been triggered during the dynamic displacement process, which is responsible for the water-wet rock before the displacement to be oil-wet during the displacement process. The difference between the dynamic and the steady relative permeabilities becomes obvious, and the dynamic effect in capillary pressure cannot be neglected when the permeability reaches ultra-low. The dynamic coefficient can reveal the shape of the displacement front.
\end{abstract}

\section{Introduction}

Low to tight permeability oil and gas reservoirs have been an important part of all the petroleum reserves due to the depletion of conventional petroleum reserves (Wang et al., 2017; Hu et al., 2018). Water flooding is commonly utilized to exploit ultra-low permeability sandstone reservoirs (permeability 0.1-50 mD) (Zhang et al., 2016; Cheng et al., 2018), during which the capillary pressure is primary to predict oil recovery. The capillary pressure influences the distribution and migration of fluid (Tian et al., 2012; Cai et al., 2014; Xiao et al., 2018); therefore, the study of the capillary pressure and its corresponding fluid flow behavior can be very useful for the design and evaluation of water flooding and the improvement of the oil and gas recovery.

The relationship between the capillary pressure $\left(P_{c}\right)$ and the wetting phase saturation $\left(S_{w}\right)$ is obtained through equilibrium fluid flow laboratory experiments routinely, where the change rate of fluid saturation $\left(\partial S_{w} / \partial t\right)$ is close to 0 (Barenblatt et al., 2003; Li et al., 2016). However, if the porous media is poorly permeable or strongly heterogeneous, establishment of the equilibrium can be time-consuming and unrealistic, and the multiphase flow is non-equilibrium at the most time ( $\mathrm{O}^{\prime}$ Carroll et al., 2005; Mumford and $\mathrm{O}^{\prime}$ Carroll, 2011; Das and Mirzaei, 2012). In these non-equilibrium situations, the relationship between capillary pressure and saturation is not only the function of fluid saturation, but also the change rate of fluid saturation, capillary numbers, et al., which is called the dynamic effect (or non-equilibrium effect) (Wang et al., 2017; Tang et al., 2018). As illustrated in Fig. 1a, the dynamic effect causes the difference between the dynamic

*Corresponding author.

E-mail address: yingingli@163.com (Y. Li); lihaitao@swpu.edu.cn (H. Li); caijc@cug.edu.cn (J. Cai); regisma@163.com (Q. Ma);

350147048@qq.com (J. Zhang).

2652-3310 (c) The Author(s) 2018.

Received May 20, 2018; revised June 13, 2018; accepted June 17, 2018; available online June 24, 2018. 


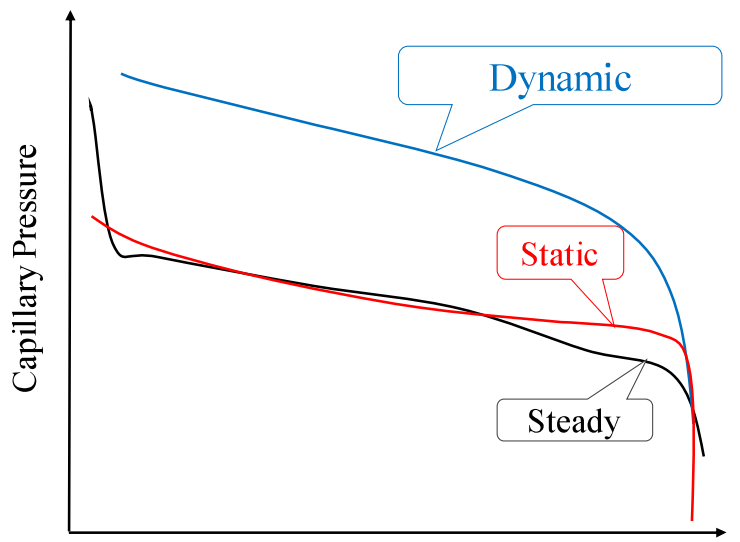

Wetting phase saturation

(a)

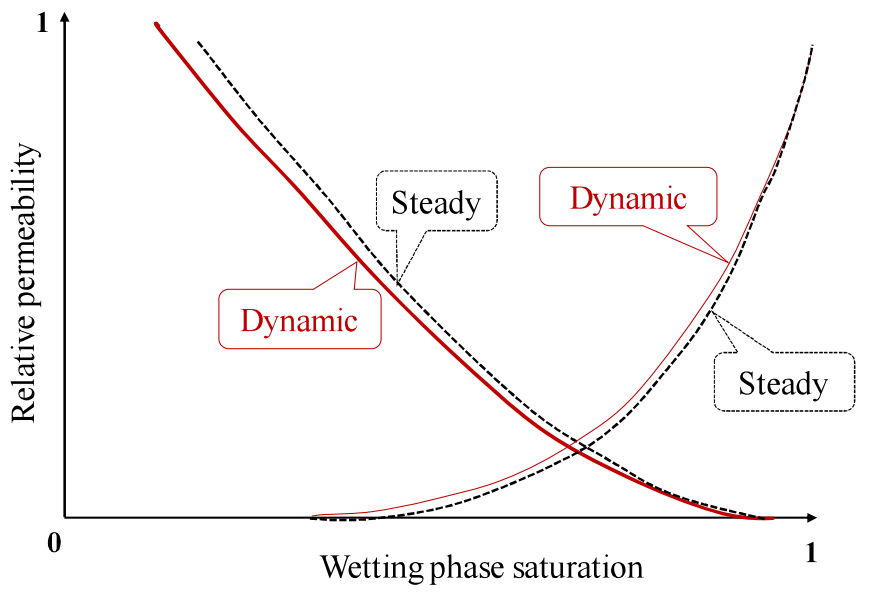

(b)

Fig. 1. The dynamic and equilibrium capillary pressures (from Topp et al., 1967).

and static capillary pressure when measuring the capillary pressure through mercury injection method and the semipermeable porous plate method (Topp et al., 1967); and the dynamic effect generates the difference between the dynamic and steady capillary pressure, as well as the difference between the dynamic and steady relative permeability in Fig. $1 \mathrm{~b}$ when fluid flowing in porous media (Mirzaei and Das, 2007; Bottero et al., 2011; Geol et al., 2016). The steady and static capillary pressure are all equilibrium capillary pressures.

Pioneered early work has proposed several models to quantitatively describe the dynamic effect in capillary pressure (Dahle and Celia, 2005; Wang et al., 2017), and a widely used model has been proposed by Hassanizadeh and Gray (1990) as:

$$
P_{c}^{d}=P_{n w}-P_{w}=P_{c}^{s}-\tau\left(\partial S_{w} / \partial t\right)
$$

where $P_{c}^{d}$ represents the dynamic capillary pressure, MPa; $P_{c}^{s}$ is the equilibrium capillary pressure, $\mathrm{MPa} ; \partial S_{w} / \partial t$ is the change rate of the wetting fluid phase saturation, $s^{-1} ; \tau$ is the dynamic coefficient, Pa.s; $P_{n w}$ and $P_{w}$ denote the non-wetting and wetting phase pressure, respectively, MPa. The dynamic effect is measured directly by the dynamic coefficient, $\tau$, Pa.s. A large dynamic coefficient exhibits that the disturbed equilibrium needs a long time to re-establish equilibrium.

In the equilibrium displacement process the capillary pressure is steady, while in the non-equilibrium displacement process the capillary pressure is dynamic. If the disturbance in the fluid flow system is eliminated quickly, the dynamic coefficient is nearly 0 and the difference between the dynamic and steady capillary pressures can be ignored. Thus, steady capillary pressure can be applied to describe a dynamic fluid flow system. Such simplification can reduce the measurement difficulty because it is much more difficult to measure the dynamic capillary pressure than the steady capillary pressure. However, this simplification is unacceptable in ultra-low permeability reservoirs because the dynamic effect can be very strong in the ultra-low permeability porous media. Therefore, the waterflooding process in ultra-low permeability reservoirs should be described by the dynamic capillary pressure and the dynamic relative permeability.

At present, studies regarding the dynamic effect in capillary pressure and the associated non-Darcy flow and fluid flow behavior remain few (Tian et al., 2012; Li et al., 2016). In this work, we examine the dynamic effect in capillary pressure and the associated fluid flow behaviors during displacement processes in ultra-low permeability reservoirs. This work provides theoretical guidance for high recovery efficiency waterflooding in ultra-low permeability reservoirs.

\section{Material and methods}

\subsection{Materials}

\section{(1) Core samples}

The experimental rock samples selected in this work are ultra-low permeability sandstone core samples collected from an ultra-low permeability sandstone formation, of which the average formation pressure is $13 \mathrm{MPa}$ and the average temperature is $57^{\circ} \mathrm{C}$. The average contact angle of the rock samples is $39.4^{\circ}$, demonstrating that the rock samples are water-wet.

Sixteen core samples were selected for the experimental tests, with diameters around $38 \mathrm{~mm}$, length around $30-35 \mathrm{~mm}$ (to meet the interval length of the test points and also to minimize the core length). Permeability and porosity of the rock samples were both measured under the in-situ condition of the formation. The permeabilities of the rock samples were measured to be $0.1-0.6 \mathrm{mD}$, with an average permeability of $0.297 \mathrm{mD}$; the porosities of the rocks samples were measured to be $2-18 \%$, with an average porosity of $8.79 \%$.

(2) Fluids

The viscosity of the formation oil is $1.21 \mathrm{mPa} \cdot \mathrm{s}$ at the formation temperature under atmospheric pressure. The viscosity of the formation brine is $0.597 \mathrm{mPa} \cdot \mathrm{s}$. The displacement water is $\mathrm{KCl}$ with a salinity of $40 \mathrm{~g} / \mathrm{L}$, which shows the same electrical resistivity with the formation brine. Thus, the water saturation can be measured by the electrical resistivity during the displacement process. In order to test the influence 
of displacement water on the expansion and contraction of rock clay minerals, the core samples were first washed, dried and measured porosity and permeability, and then were vacuumed and saturated with the displacement water for $24 \mathrm{~h}$. The porosity and permeability of the core samples were measured after cleaning and drying again. The result shows that the core physical properties are basically unchanged with the displacement water, so it is confirmed that the displacement water has little effect on clay minerals.

\subsection{Apparatus}

The apparatus (Fig. 2) is specially designed to investigate the dynamic effect in capillary pressure and the fluid flow behavior during the displacement process in low to tight porous media under high pressure and high temperature ( $\mathrm{Li}$ et al., 2019). The vital units are the pressure measurement unit and the fluid saturation measurement unit. There are two pairs of pressure transducers (PTs) for the pressure measurement unit and three pairs of LCR meters (type TH2810D) for the fluid saturation measurement unit. Semi-permeable plates (ceramic, $1500 \mathrm{psi}$ ) durable enough to bear the high imposed pressure are fixed in the pressure transducers. The LCR meter measures the electrical resistivity of the column, with an accuracy of measuring the phase saturation to be $1 \%$.

At present, the pressure transducers in the previous studies (Oung et al., 2000, 2003, 2005) can reach only up to approximately 0.07 MPa except for the study (Tian et al., 2012). This work extends the test scope to around $7 \mathrm{MPa}$, which is acceptable for ultra-low permeability porous media, and the pressure measurement does not affect the oil and water flow. In addition, the apparatus of this study consumes less semipermeable plates and is easy to be installed. The tested core column should be around $38 \mathrm{~mm}$ in diameter, longer than 30 $\mathrm{mm}$.

\subsection{Experimental Procedures}

The experimental procedures are the same as our previously published papers (Li et al., 2016, 2017, 2019). The original water and oil saturations were firstly achieved, and the experimental rig was prepared by vacuuming and saturating the semi-permeable plates. The in-situ condition was established by increasing the confining pressure, back pressure, and temperature. Then the dynamic displacement and steady displacement tests were performed.

\subsection{Experimental Methodologies}

The process of the experimental data requires strong skills to obtain fluid saturations, the steady capillary pressure, the steady relative permeability curves, the dynamic capillary pressure, and the dynamic relative permeability curves, because the samples are ultra-low permeability. Special data processing methods are required to calculate water saturation, and the measured data of the PTs should be calibrated. The $\tau-S_{w}$ relationship can be obtained from the dynamic capillary pressure-saturation relationship, the steady capillary pressure-

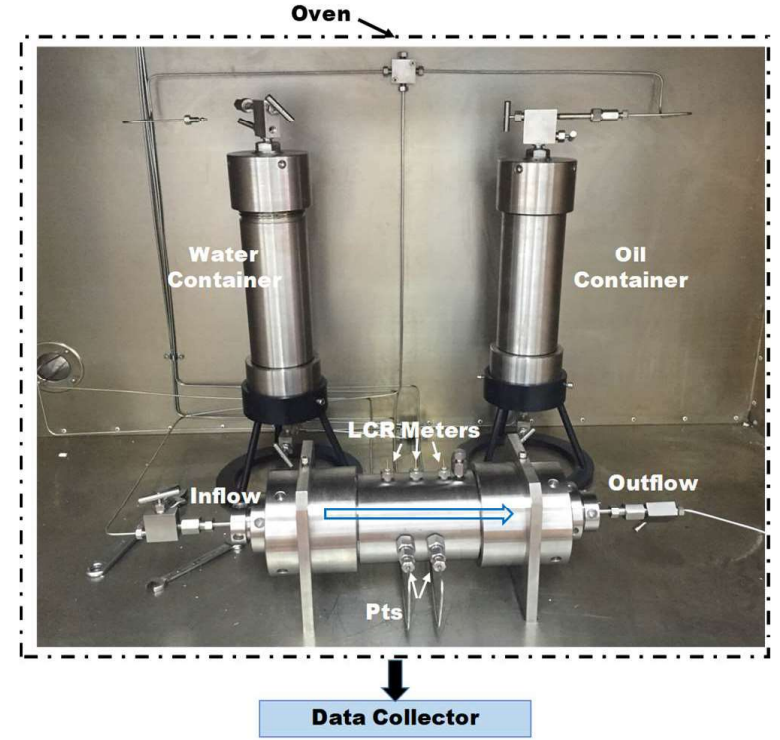

Fig. 2. The experimental apparatus to measure the dynamic effect.

saturation relationship and the time derivative of saturation measured by the experiment. The experimental methodologies are the same as our previous work, which has been illustrated carefully ( $\mathrm{Li}$ et al., 2017, 2019). Note that the response characteristics of the sensors can affect the read of the PTs, so the in-situ single-phase pressure was corrected according to Hou et al. (2012).

\section{Results and discussion}

Six less permeable core samples experienced very long displacement time during the experimental process, and the change rate of water saturations was very low, with the recovery rate no more than $20 \%$. In the steady displacement experiments, the change rates of water saturations were very slow within $12 \mathrm{~h}$ for these six core samples, and only a few oils flowed out, making it difficult to measure the oil volume. The reasons may be that the pores are too tight and the initially established oil saturations did not reach the expected values, or the displacement pressure is too low to allow the fluid flow. Therefore, these six core samples were not used as effective experimental data sources.

\subsection{Dynamic capillary pressure-saturation curves}

Typical dynamic and steady capillary pressures are depicted in Fig. 3. It can be seen that the water phase pressure is higher than the oil phase pressure at the early stage of the steady displacement process, and is lower than the oil phase pressure at the later stage. However, the water phase pressure is always lower than the oil phase pressure during the dynamic displacement process. This indicates that the steady capillary pressure is the driving forces and then becomes the resistance, but the dynamic capillary pressure is the resistance during all the dynamic displacement process. 


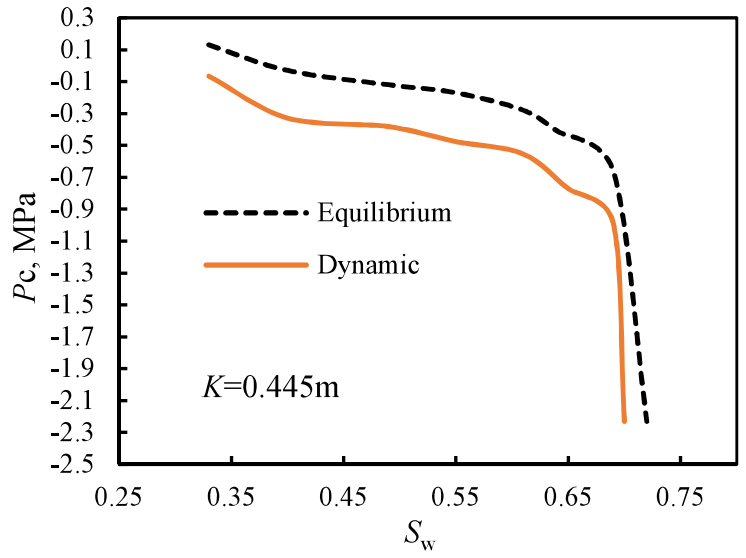

(a)

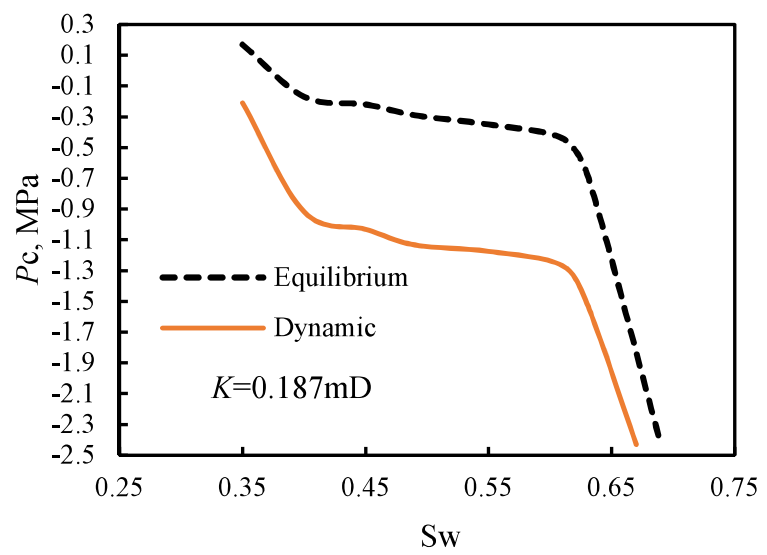

(c)

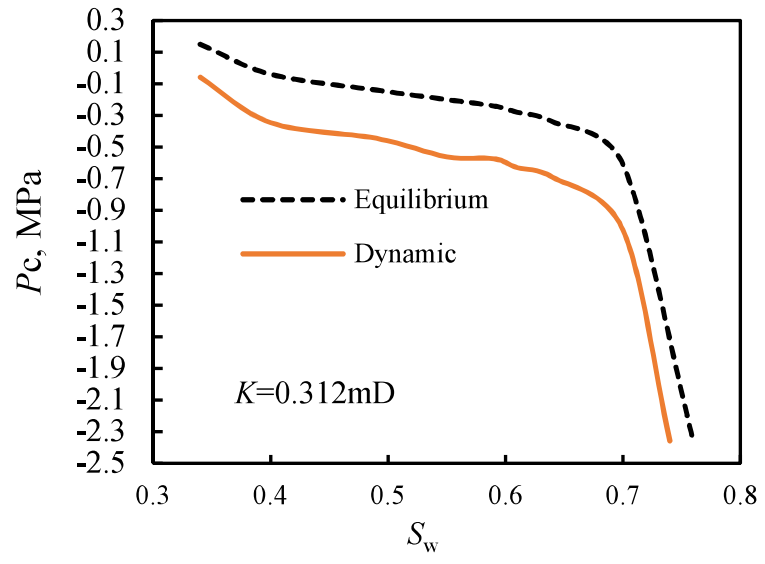

(b)

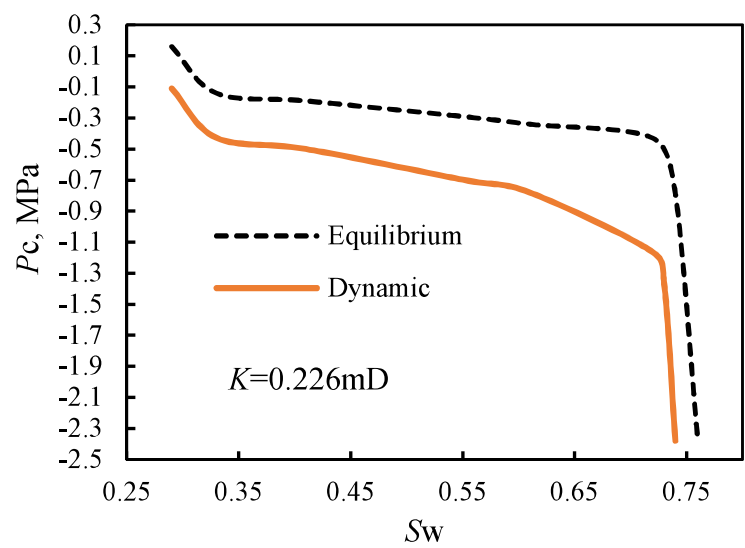

(d)

Fig. 3. Capillary pressure during the displacement in ultra-low permeability sandstone reservoirs.

The dynamic capillary pressures reverse more than the steady ones, which shows that the dynamic capillary pressures impede the fluid flow more strongly than the steady ones during the displacement process. The distinction between the dynamic and the steady capillary pressure becomes more significant when the permeability is lower under the same displacement pressure.

The behaviors of capillary pressures demonstrate that wettability alteration has been triggered during the displacement process. The originally water-wet rock seems to be oil-wet after a short time since the displacement begins. The displacement of water to oil is a forced imbibition process rather spontaneous imbibition. This is constant with the previous study (Song and Zhuo, 2009; Wang et al., 2009).

The contact angle in this study is calculated assuming that the minimal pore radius allowing fluid flow is $20 \mathrm{~nm}$ (Xiao, 2015). The dynamic contact angles during the dynamic displacement process in ultra-low permeability rock are illustrated in Fig. 4. The dynamic contact angles are increasing with the displacement process, which shows that wettability alteration follows the whole displacement process. In addition, the dynamic contact angles of the same pore size increase with the decrease of permeability. This shows that for the same pore

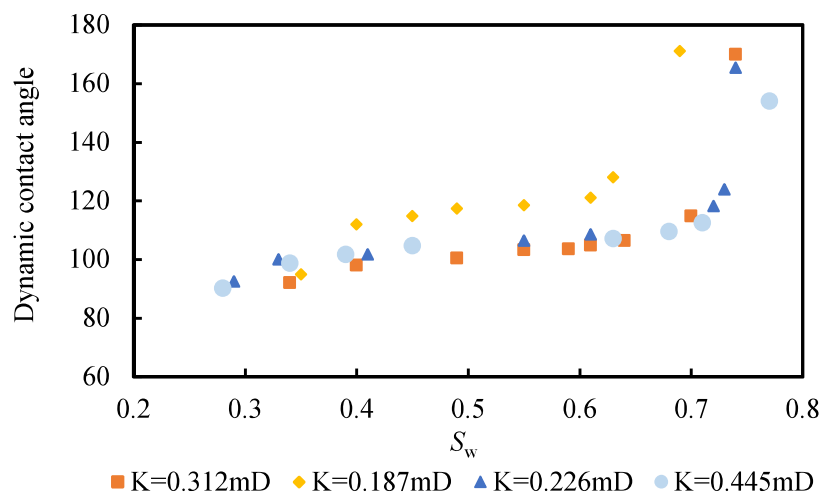

Fig. 4. Dynamic contact angle.

sizes, pores in the lower permeability rock experience higher displacement pressure than that in the higher permeability rock. Thus, the dynamic contact angles in the lower permeability rock are larger than that in the higher permeability rock, and wettability alteration tends to be triggered to a larger degree. This generates higher capillary resistance. 


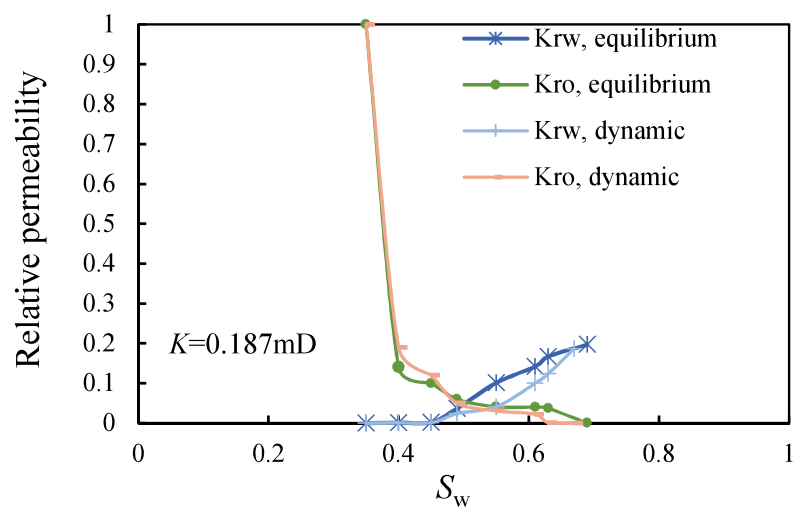

(a)

Fig. 5. The dynamic and the steady relative permeability curves.

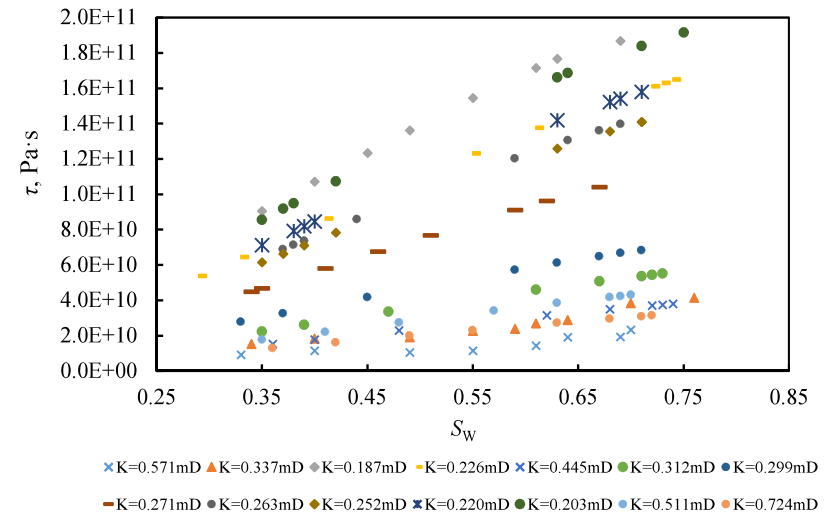

Fig. 6. The dynamic coefficient of the matrix.

\subsection{Relative permeability}

The multiphase flow can be refleted by the dynamic relative permeability in ultra-low permeability sandstone reservoirs. Representative oil and water relative permeability curves in the ultra-low permeability sandstone reservoirs are depicted in Fig. 5. The dynamic oil relative permeability curves are slightly higher than the steady ones when the water saturation is low, but soon lower than the steady ones after the water saturation exceeds a certain value. The dynamic water relative permeability curves are slightly higher than that of the steady ones when water saturation is low, and are slightly lower than that the steady water relative permeability curves after water saturation increases.

The dynamic relative permeability curves of ultra-low permeability sandstone are different from those of the medium to high permeability rocks and even low permeability rocks. The dynamic oil relative permeability of medium to high permeability rocks is lower than the steady oil relative permeability, which experiences no time period to be higher than the steady oil relative permeability. This is because the sizes of pores in ultra-low permeability sandstone are smaller than medium to high permeability rocks, so higher displacement pressures are needed to displacement the fluid in these tiny

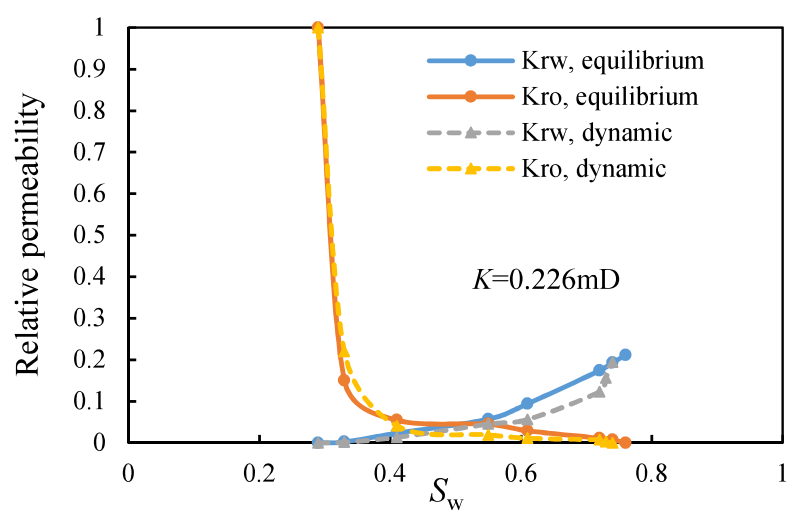

(b)

pores. Thus, the fluids flow faster at the early stage during the dynamic displacement process than that during the steady displacement process since the displacement pressures of the dynamic displacement process are higher than that of the steady displacement process. However, the displacement water contacts more with the rock wall during the steady displacement process than that during the dynamic displacement process, and thus the displacement water during the steady displacement process connects more with the confined water, peeling off more oil. This is the reason why the steady oil and water relative permeability curves are higher than the dynamic oil and water relative permeability curves.

\subsection{Dynamic effect}

The relationships between the dynamic coefficients and water saturations of the fourteen core samples are compared in Fig. 6. It is observed that briefly, the dynamic coefficients increase nearly exponentially with the increase of the water saturation. Dahle et al. (2005) also reported that the dynamic coefficient decreases with the decrease of wetting phase saturations. This results from that water enter the large pores initially during the displacement process, and then into the smaller pores. Since the dynamic effects in the larger pores are weaker than that in the smaller pores, the dynamic coefficient rises along with the entrance of water from the larger pores to the smaller pores. In conclusion, the dynamic effect reflects the uniformity of fluid flow in pores during the displacement process. With the same fluid properties, the dynamic coefficient is affected mainly by the pore structures. If the dynamic coefficient fluctuates more profoundly, the fluid flows more non-uniformly with a stronger fingered flow.

The lower the permeability of the rocks, the higher the dynamic coefficient, and the stronger the dynamic effect in capillary pressure. This trend is similar to the results of Stauffer (1978) and Das (2007). This is because that as the permeability decreases, the pore heterogeneity increases and the pore size decreases.

Further experiments were conducted on thirty medium to low permeability $(1 \sim 200 \mathrm{mD})$ core samples to expand the re- 
Table 1. The dynamic coefficient of the different permeability rock.

\begin{tabular}{lllll}
\hline Permeability $(\mathrm{mD})$ & $<1$ & $1 \sim 10$ & $10 \sim 50$ & $50 \sim 200$ \\
\hline Dynamic coefficient $(\mathrm{Pa} \cdot \mathrm{s})$ & $10^{9} \sim 10^{11}$ & $10^{8} \sim 10^{9}$ & $10^{7} \sim 10^{8}$ & $10^{6} \sim 10^{7}$ \\
\hline
\end{tabular}

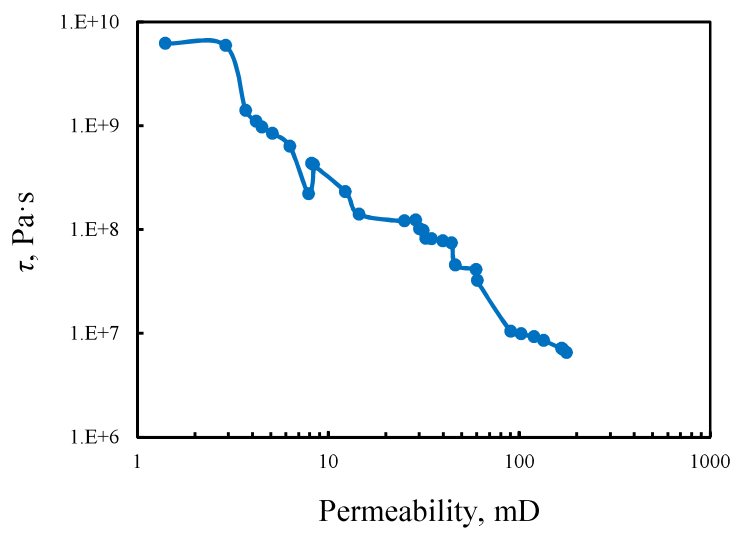

Fig. 7. The dynamic coefficient of the different permeability rock.

lationship between the dynamic coefficient and the permeability. As provided in Fig. 7 and Table 1, generally the dynamic coefficient increases with the decrease of permeability. The dynamic coefficient of the target ultra-low permeability sandstone reservoir is in the range of $10^{9} \sim 10^{11} \mathrm{~Pa} \cdot \mathrm{s}$. Such value is much larger than that of high to medium porous media (smaller than $\left.10^{8} \mathrm{~Pa} \cdot \mathrm{s}\right)$. The dynamic coefficient reported by Zhang et al. (2013) and Jia and lv (2014) can reach up to $10^{13} \mathrm{~Pa} \cdot \mathrm{s}$, which is even higher than that of this study. Therefore, the dynamic effect in capillary pressure can be so significant that should be involved in the evaluation and recovery prediction of reservoir.

\subsection{Change rate of fluid saturation}

It has been concluded that the dynamic coefficient can reflect the uniformity of fluid flow in pores during the displacement process, which can be confirmed by the change rates of fluid saturations shown in Fig. 8. The change rate of water saturation jumps quickly and then declines slowly along with the increase in water saturation. This is due to that the flowing resistances are increasing when water enters the smaller pores from the initially larger pores.

\subsection{The critical condition to consider the dynamic effect}

As the relationship between the dynamic coefficient and permeability illustrated in Fig. 9, the dynamic coefficient can be larger than $10^{8} \mathrm{~Pa} \cdot \mathrm{s}$ and rises fast when permeability is lower than $15 \mathrm{mD}$. Moreover, the previous study (Zhang et al., 2015) has reported that the difference between the dynamic and steady capillary pressure can be larger than $0.1 \mathrm{MPa}$ and increases along with the decrease of the average pore radius,

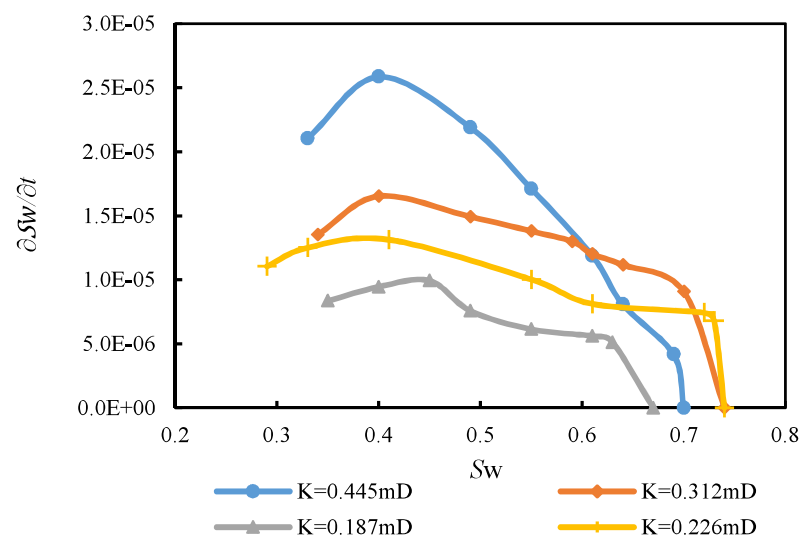

Fig. 8. Change rate of water saturation.

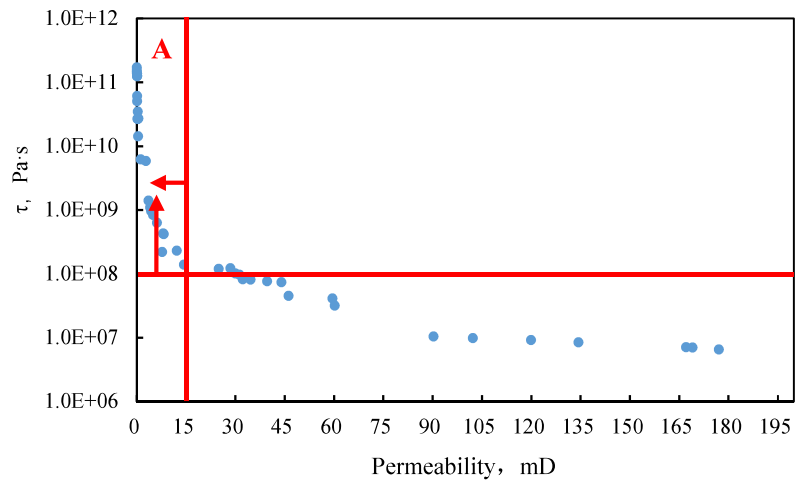

Fig. 9. The relationship between the dynamic effect and permeability.

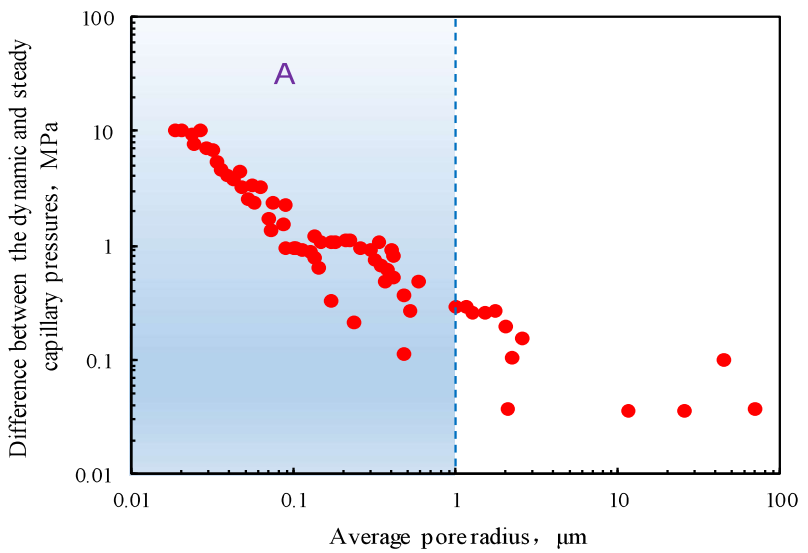

Fig. 10. Difference between the dynamic and the steady capillary pressures with the average pore radius (from Zhang et al., 2015).

as shown in Fig. 10. Therefore, this study proposed that the critical condition to consider the dynamic effect is: when the 
permeability is lower than $15 \mathrm{mD}$, the dynamic coefficient is larger than $10^{8} \mathrm{~Pa} \cdot \mathrm{s}$, and the average pore radius is smaller than $1 \mu \mathrm{m}$, which is the A area described in Fig. 9 and Fig. 10.

\section{Conclusions}

This paper investigates the characteristics and mechanisms of dynamic effect in capillary pressure during the waterflooding process in ultra-low permeability sandstone reservoirs through self-developed apparatus and methods. The critical condition to consider the dynamic effect in capillary pressure is proposed. The experimental tests and theoretical analyses contribute to the following conclusions:

(1) The dynamic effect in capillary pressure is stronger in a lower permeability porous media. The dynamic effect in ultralow permeability reservoirs will cause a noticeable difference between the dynamic and the steady relative permeabilities, which should be involved in the reservoir evaluation and development.

(2) Wettability alteration has been triggered during the displacement process. The originally water-wet rock seems to be oil-wet after a short time since the displacement begins. The displacement of water to oil is a forced imbibition process rather spontaneous imbibition.

(3) The dynamic effect reflects the uniformity of fluid flow in pores during the displacement process. If the dynamic coefficient fluctuates more profoundly, the fluid flows more non-uniformly with a stronger fingered flow.

(4) The critical condition to consider the dynamic effect is: when the permeability is lower than $15 \mathrm{mD}$, the dynamic coefficient is larger than $10^{8} \mathrm{~Pa} \cdot \mathrm{s}$, and the average pore radius is smaller than $1 \mu \mathrm{m}$.

\section{Acknowledgement}

This work was supported by the National Natural Science Foundation of China (No. 41902161) and the China Postdoctoral Science Foundation (No. 2018M643526).

\section{Conflict of interest}

The authors declare no competing interest.

Open Access This article is distributed under the terms and conditions of the Creative Commons Attribution (CC BY-NC-ND) license, which permits unrestricted use, distribution, and reproduction in any medium, provided the original work is properly cited.

\section{References}

Barenblatt, G.I., Patzek, T.W., Silin, D.B. The mathematical model of nonequilibrium effects in water-oil displacement. SPE J. 2003, 8(4): 409-416.

Bottero, S., Hassanizadeh, S.M., Kleingeld, P.J., et al. Nonequilibrium capillarity effects in two-phase flow through porous media at different scales. Water Resour. Res. 2011, 47(10): W10505.

Cai, J., Perfect, E., Cheng, C., et al. Generalized modeling of spontaneous imbibition based on Hagen-Poiseuille flow in tortuous capillaries with variably shaped apertures. Langmuir 2014, 30(18): 5142-5151.

Cheng, S., Li, M., He, Y., et al. A multi-well interference pressure transient analysis method to determine the water source orientation of multi-fractured horizontal well. Journal of China University of Petroleum (Edition of Natural Science) 2018, 42(5): 81-88.

Dahle, H.K., Celia, M.A., Hassanizadeh, S.M. Bundle-oftubes model for calculating dynamic effects in the capillary-pressure-saturation relationship. Trans. Porous Med. 2005, 58(1-2): 5-22.

Das, D.B., Mirzaei, M. Dynamic effects in capillary pressure relationships for two-phase flow in porous media: Experiments and numerical analyses. AIChE J. 2012, 58(12): 3891-3903.

Hassanizadeh, S.M., Gray, W.G. Mechanics and thermodynamics of multiphase flow in porous media including interphase boundaries. Adv. Water Resour. 1990, 13(4): 169-186.

Hou, L., Chen, L., Kibbey, T.C.G. Dynamic capillary effects in a small-volume unsaturated porous medium: Implications of sensor response and gas pressure gradients for understanding system dependencies. Water Resour. Res. 2012, 48(11): W11522.

$\mathrm{Hu}, \mathrm{W}$., Wei, Y., Bao, J. Development of the theory and technology for low permeability reservoirs in China. Petrol. Explor. Develop. 2018, 45(4): 646-656.

Jia, A., Lv, Z. Dynamic Effect of capillary pressure in tight gas reservoir. Open Petro. Eng. J. 2014, 7(1): 71-79.

Li, A., Fu, S., Zhang, H., et al. A capillary pressure measurement method at real reservoir conditions. Journal of China University of Petroleum (Edition of Natural Science), 2016, 40(3):102-106.

Li, H., Li, Y., Chen, S., et al. Effects of chemical additives on dynamic capillary pressure during waterflooding in low permeability reservoirs. Energ. Fuel 2016, 30(9): 70827093.

Li, Y., Li, H., Chen, S., et al. Capillarity characters measurement and effects analysis in different permeability formations during waterflooding. Fuel 2017, 194: 129143.

Li, Y., Li, H., Chen, S., et al. The second critical capillary number for chemical flooding in low permeability reservoirs: Experimental and numerical investigations. Chem. Eng. Sci. 2019, 196: 202-213.

Mirzaei, M., Das, D.B. Dynamic effects in capillary pressure-saturations relationships for two-phase flow in 3D porous media: Implications of micro-heterogeneities. Chem. Eng. Sci. 2007, 62(7): 1927-1947.

Mumford, K.G., O'Carroll, D.M. Drainage under nonequilibrium conditions: Exploring wettability and dynamic contact angle effects using bundle-of-tubes simulations. Vadose Zone J. 2011, 10(4): 1162-1172.

O'Carroll, D.M., Phelan, T.J., Abriola, L.M. Exploring dynamic effects in capillary pressure in multistep outflow experiments. Water Resour. Res. 2005, 41(11): W1419.

Oung, O., Bezuijen, A. Selective pore pressure transducers for use in model tests to study two-phase flow in porous 
media. Int. J. Phys. Model. Geotech. 2003, 3(4): 31-41.

Oung, O., Schenkeveld, F.M., Weststrate, F. Centrifuge research on the transport of DNAPLs: The use of dielectric water content sensor to study DNAPL transport in porous media in centrifuge experiments. International Symposium on Physical Modelling and Testing in Environmental Geothenics. La Baule, France, 2000, 317324.

Oung, O., Hassanizadeh, S.M., Bezuijen, A. Two-phase flow experiments in a geocentrifuge and the significance of dynamic capillary pressure effect. J. Porous Med. 2005, 8(3): 247-257.

Song, F., Zhuo, J. Movable interface between two fluids in quartz micro-channels. Chinese J. Appl. Mech. 2009, 26(4): 633-637.

Stauffer, F. Time dependence of the relations between capillary pressure, water content and conductivity during drainage of porous media. IAHR symposium on scale effects in porous media, Thessaloniki, Greece, 1978, 29: 3-35.

Tang, M., Lu, S., Zhan, H., et al. The effect of a microscale fracture on dynamic capillary pressure of two phase flow in porous media. Adv. Water Resour. 2018, 113: 272-284.

Tian, S., Lei, G., He, S., et al. Dynamic effect of capillary pressure in low permeability reservoirs. Petrol. Explor. Develop. 2012, 3(39): 378-384.

Wang, L., Tian, Y., Yu, X., et al. Advances in improved/enhanced oil recovery technologies for tight and shale reservoirs (Review). Fuel 2017, 210: 425-445.
Wang, S.L., Liu, P.C., Zhao, H., et al. A novel method for calculating the dynamic capillary force and correcting the pressure error in micro-tube experiment. Sci. Rep. 2017, 7: 16590.

Wang, Z., Zhou, W., Dong, J., et al. The change of dynamic capillary pressure during water oil displacement in micron-scale capillary. Proceedings of the 12th conference on colloids and interfacial chemistry of the Chinese chemical society, 2009.

Xiao, J., Cai, J., Xu J. Saturated imbibition under the influence of gravity and geometry. J. Colloid Interf. Sci. 2018, 521: 226-231.

Xiao, Q. The reservoir evaluation and porous flow mechanism for typical tight oilfields. University of Chinese Acedemy of Sciences (Institute of Porous Flow \& Fluid Mechanics), 2015.

Zhang, H., He, S., Jiao, C., et al. Study of dynamic capillary pressure in ultra-low permeability reservoir. Sci. Technol. Eng. 2013, 13(12): 3261-3266.

Zhang, H., He, S., Jiao, C., et al. Investigation of dynamic effect of capillary pressure in ultra-low permeability sandstones. Indian Geotech. J. 2015, 45(1): 79-88.

Zhang, Z., Shi, Y., Li, H. Stress sensitivity anisotropy and its microscopic mechanism of tight oil and gas reservoirs: A case from the Chang-6 reservoir in Ansai oilfield of Ordos Basin. Oil Gas Geolog. 2016, 37(1): 117-124. 